\title{
Modeling Transfer Function of Electrical Power Lines for Broadband Power Line Communication
}

\author{
Saad D. Bennani \\ Ecole Nationale des Sciences Appliquées, Fès, Marocco \\ Email: saad.d.bennani@gmail.com
}

Received September 29, 2011; revised November 23, 2011; accepted December 6, 2011

\begin{abstract}
The indoor access network is characterized by several branches that cause many reflections. In this paper, we present an approach to model the transfer function of electrical power lines for broadband power line communication. The assuming channel consists of three wires, which is widely used in many countries. As an application of the proposed method, the effect of line length from transmitter to receiver, branched line length and number of branched are also studied. From the frequency response of the transfer function (magnitude and phase), it is seen that the position of notches and peaks in the magnitude responses are largely affected in terms of attenuation by the electrical network which consists of 15 branches.
\end{abstract}

Keywords: Power Line Communications; Transmission Line Theory; Magnitude Response and Branched Line

\section{Introduction}

The power-line communications (PLC) has been applied as a data transfer method in both public electricity distribution networks and indoor distribution networks. The profile of these applications has been different. The devices developed for domestic use are mainly designed for the purpose of controlling electric devices at home. For example, the current and most common functions (Figure 1) are: control of lights, fire alarming system and heating controlling [1].

The design of an appropriate communication system, the choice of modulation and coding schemes, and the evaluation of performance, require the knowledge of the end-to-end PLC channel responses in a wide range of frequencies up to $75 \mathrm{MHz}$ [2].

Several approaches for modelling the transfer characteristics of power lines can be found in literature. The most widely used model for the power line channel transfer function $H(f)$ is the multipath model proposed by Philipps [3] and Zimmermann [4].

Actually, the proposed model is the result of a topdown approach: the various parameters need to be determined through measurements. As such, it is not suitable for the computation at first of the actual transfer function of a given link. Alternative models, based on the multiconductor transmission line theory (MTL), have been proposed in the case of indoor channels [2-5].

The objective of this paper is to calculate the transfer function for a power line communications channel which consists on fifty branches. As an application of the proposed method, the effect of line length from transmitter to receiver, branched line length and number of branched are also studied.

\section{Influence of Line Length}

\subsection{Length from Transmitter to the Receiver}

In this part, we are going to see the influence of line length on response of the PLC channel.

We consider a typical medium voltage line of a power line communications which consists of fifteen branches. The PLC network and all branches are formed by three conductors. All the lines have the following per unit length parameters [6] with:

$$
\begin{gathered}
R=\sqrt{\frac{2 \mu_{r} \mu_{0} f}{\pi \sigma r^{2}}}\left[\frac{(D / 2 r)}{(D / 2 r)^{2}-1}\right](\Omega / \mathrm{m}) \\
L=\frac{\mu_{0} \mu_{r}}{\pi} \cosh ^{-1}\left(\frac{D}{2 r}\right)+\frac{R}{2 \pi f}(\mathrm{H} / \mathrm{m})
\end{gathered}
$$

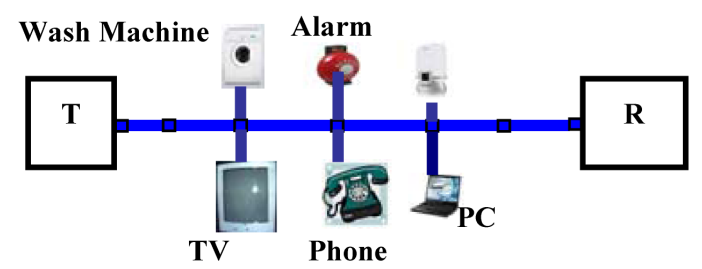

Figure 1. Model of a PLC network. 


$$
\begin{gathered}
C=\frac{\pi \cdot \varepsilon_{0} \cdot \varepsilon_{r}}{\cosh ^{-1}(D / 2 r)}(\mathrm{F} / \mathrm{m}) \\
G=2 \cdot \pi \cdot f \cdot C \cdot\left(-5.7257 \cdot 10^{-10} \cdot f+0.06\right)\left(\mathrm{S}^{-1} / \mathrm{m}\right)
\end{gathered}
$$

where, $r$ is the radius of the conductors $(r=0.8 \mathrm{~mm}), D$ is the distance between the conductors $(D=3.2 \mathrm{~mm}), \sigma$ is the electric conductivity of the conductors $(\sigma=5.5758 \times$ $\left.10^{7}\right), \mu$ is the magnetic permeability $\left(\mu=\mu_{0} \mu_{r}, \mu_{0}=\right.$ $\left.4 \pi \cdot 10^{-7} \mathrm{H} / \mathrm{m}, \mu_{r}=1\right), \varepsilon$ is the dielectric constant $\left(\varepsilon=\varepsilon_{0} \varepsilon_{r}\right.$, $\varepsilon_{0}=8.8542 \times 10^{-12} \mathrm{~F} / \mathrm{m}$ ).

The configuration under study is given in Figure 2 with $Z L=50 \Omega, Z g=50 \Omega$. The line length Transmitterreceiver was varied as $48 \mathrm{~m}, 64 \mathrm{~m}, 80 \mathrm{~m}$ and $96 \mathrm{~m}$.

Figures 3(a)-(d) shows the magnitude of the transfer function versus frequency $H(f)[6]$ relating the transmitter and the receiver for $48 \mathrm{~m}, 64 \mathrm{~m}, 80 \mathrm{~m}$ and $96 \mathrm{~m}$ respectively. From Figure 3 the peak values of transfer function were not attenuating significantly with either frequency or line length. Any differences that are seen could be attributed to the finite losses due the line series resistance. The position of notches in the transfer function of medium voltage channel also does not depend on length from transmitter to receiver.

Figures 3(a)-(d) shows the corresponding phase responses. It is observed that as the line length increases there are rapid changes in the phase response. The effects of branch length are studied next.

\subsection{Branched Length}

We now consider the configuration as given in Figure 2; i.e. the length of a line from transmitter to receiver was kept constant at $48 \mathrm{~m}$. The branched length was varied as ( $L b_{i}=2 \mathrm{~m}, 3 \mathrm{~m}, 4 \mathrm{~m}$ and $5 \mathrm{~m}$ for $i=1: 15$ branches) with the distance between every branch is constant and equal to $3 \mathrm{~m}$. The fifteen branches are terminated by open circuits.

We calculate the transfer characteristics with respect to the receiver [6]. Figure 4 show the corresponding frequency responses for various branch line lengths. It is observed that in all cases the peaks of frequency responses for various branches was not either attenuating with frequencies or branch length similar to the earlier case. Whereas, the position of the peaks and notches is case dependant unlike the previous case.

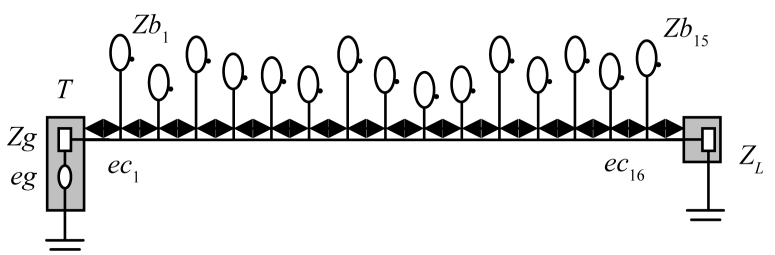

Figure 2. Power line network with 15 branches.

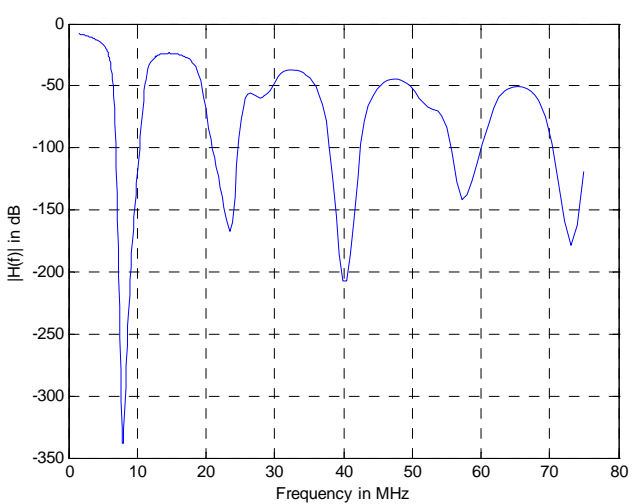

(a)

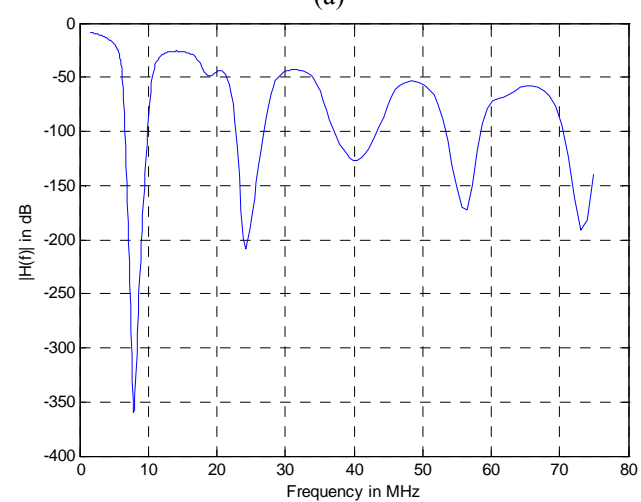

(b)

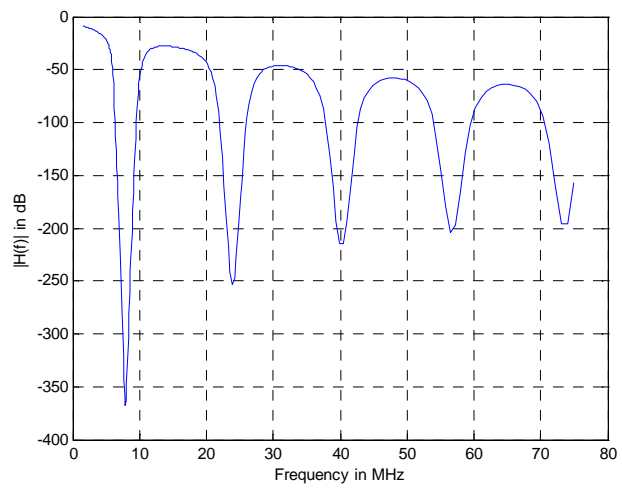

(c)

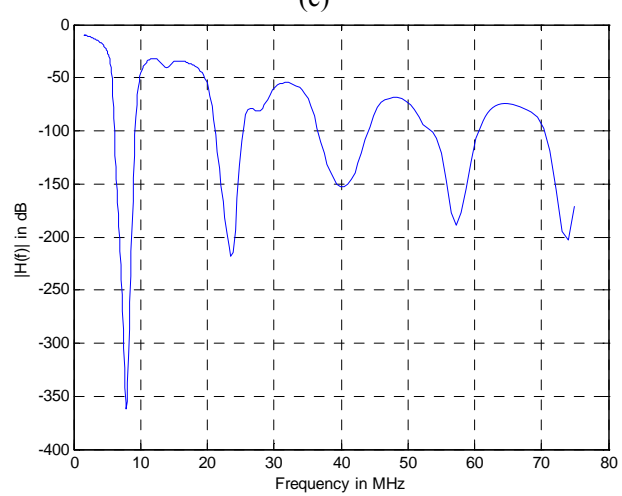

(d)

Figure 3. Simulation results for medium channel of power line link with 15 branches (a) $48 \mathrm{~m}$; (b) $64 \mathrm{~m}$; (c) $80 \mathrm{~m}$; and (d) $96 \mathrm{~m}$. 


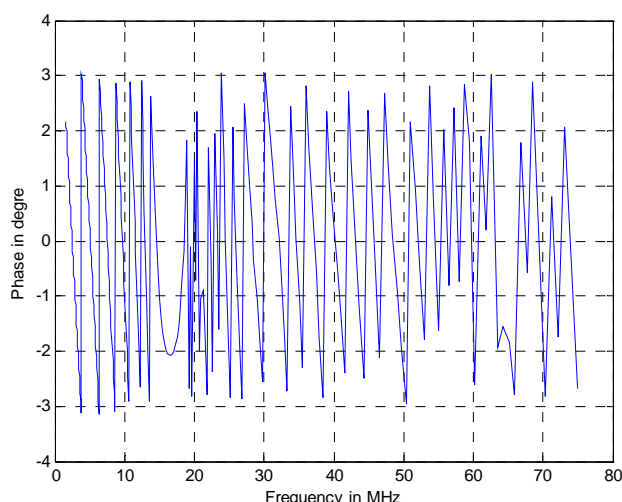

(a)

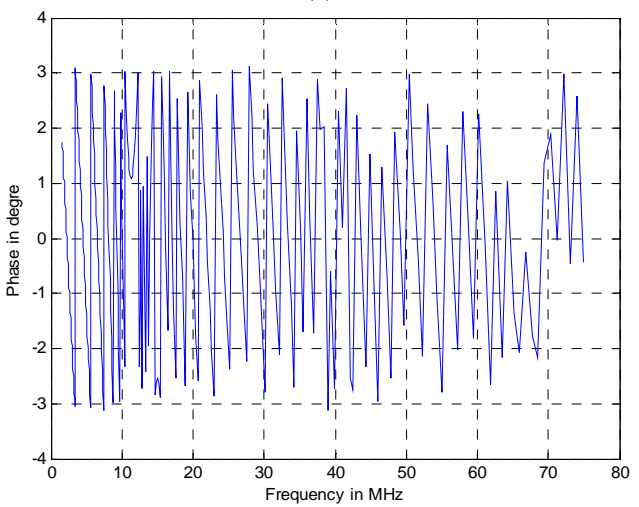

(b)

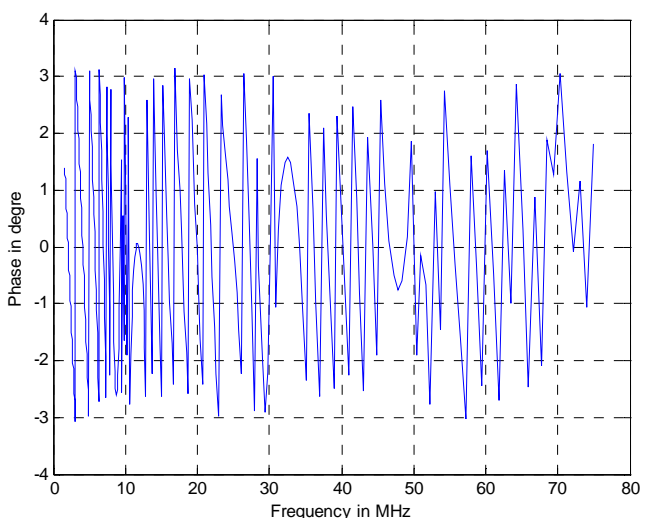

(c)

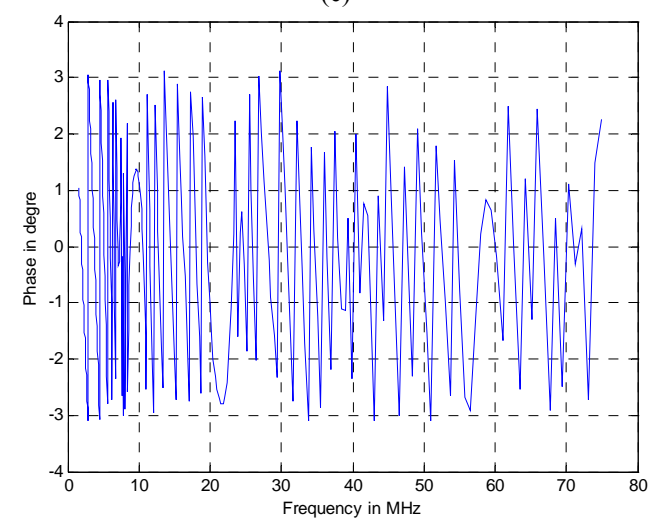

(d)

Figure 4. Phase response for PLC with 15 branches (a) 1 m; (b) $2 \mathrm{~m}$; (c) $3 \mathrm{~m}$; (d) $4 \mathrm{~m}$.
As the length of branched line increases the number of notches increase. The phase response (Figure 5) for the case under study had similar behavior as in Figure 6.

\section{Influence of Number of Branches}

We consider the power line channel with distributed branches as shown in Figure 2. The number of branches was varied in the link between transmitter and receiver. We note that the distance between transmitter and receiver was kept constant at $48 \mathrm{~m}$, while all branches were $5 \mathrm{~m}$ long. The number of branches was varied as $1,5,10$ and 15.

Note that for each case the distances between the transmitter and the receiver were equal but not equally distributed [(case $\left.1 e c_{1}=e c_{2}=24 \mathrm{~m}\right),\left(\right.$ case $2 e c_{1}=e c_{2}=e c_{3}$ $\left.=e c_{4}=e c_{5}=3 \mathrm{~m} \mathrm{ec}_{6}=33 \mathrm{~m}\right),\left(\right.$ case $3 e c_{1}=e c_{2}=e c_{3}=$ $\left.e c_{4}=e c_{5}=e c_{6}=e c_{7}=e c_{8}=e c_{9}=e c_{10}=3 \mathrm{~m} \mathrm{ec}_{11}=18 \mathrm{~m}\right)$ and (case $4 e c_{i}=3 \mathrm{mi}=1: 16$ )]. The terminations of all the branches are connected on open circuit. Figure 7 shows the corresponding frequency responses for differrent number of branches. It is observed that the positions of notches are not changed. But as the number of branches increases the attenuations of notched point tends to increase. The phase responses are comparable to previous cases as shown in Figure 8.

\section{Summary of the Conditions}

The parametric study conducted in this paper showed that the number of branches, the length of the line and length of connections leads an attenuation of the transfer function.

\section{Conclusions}

In this paper, we were interested in modeling transfer function of electrical power lines for broadband power line communication.

A detailed study of factors influencing wave propagations in the medium voltage lines was done.

The magnitude of the transfer function is reduced and selective versus the frequency.

We showed that the notches in PLC channel do not depend on the line length from the position of the transmitter and receiver. The pulse distortion does not depend on the line length between transmitter and receiver. The position of notches in frequency response depends on the branched line length. The increase in branched line length tends to limit the available bandwidth in the medium voltage channel.

We also showed that the position of deep notches does not change with a number of distributed branches. As the number of distributed branches increases the amplitude of notched points tends to increase. 


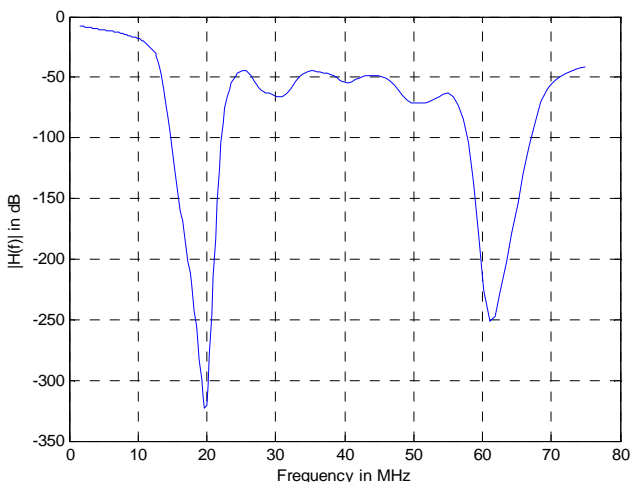

(a)

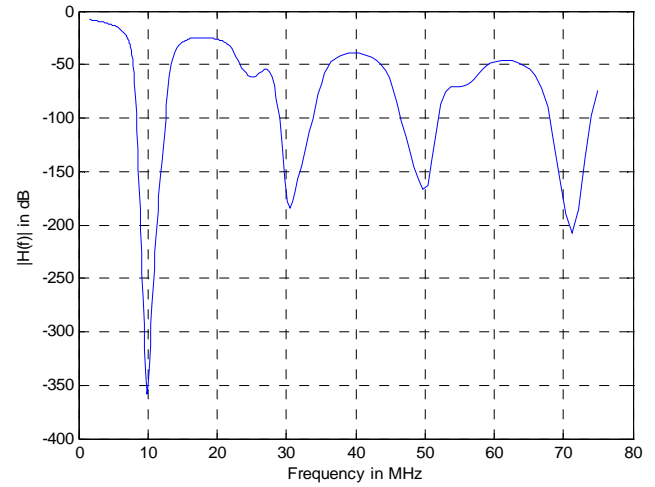

(c)

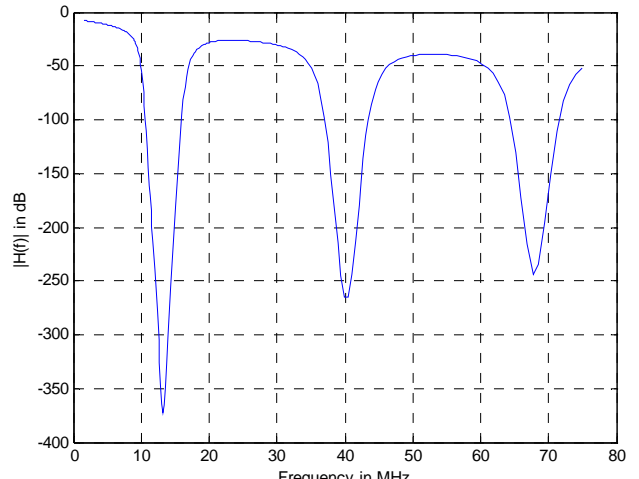

(b)

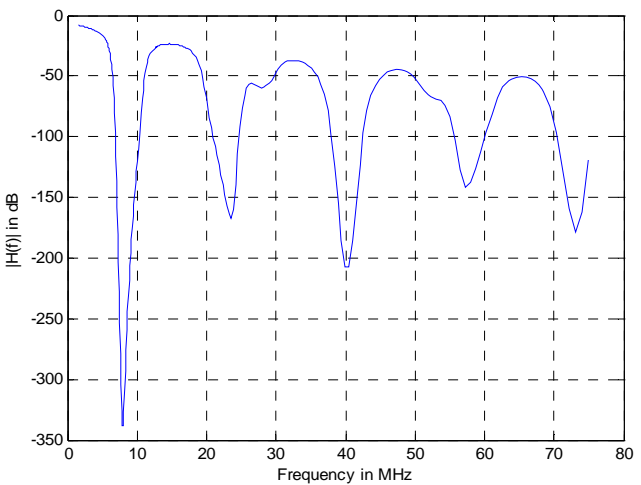

(d)

Figure 5. Simulation results for PLC with 15 branches (a) 1 m; (b) 2 m; (c) 3 m; (d) 4 m.

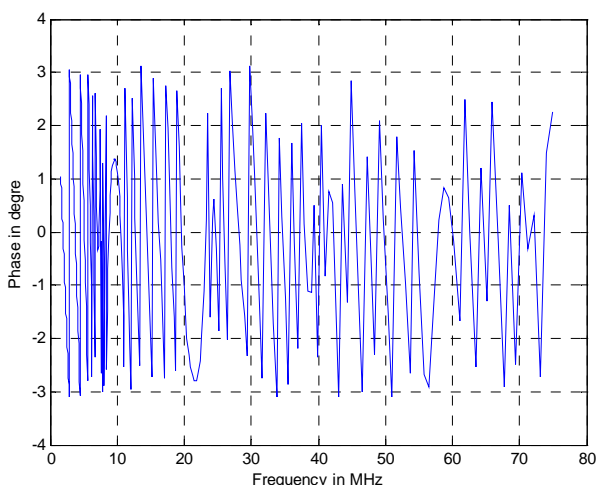

(a)

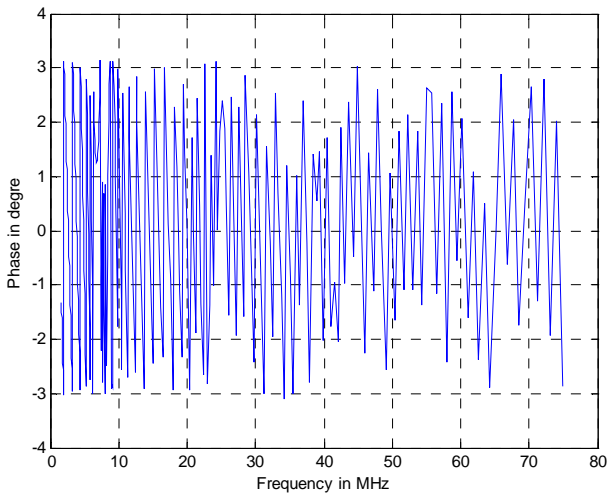

(c)

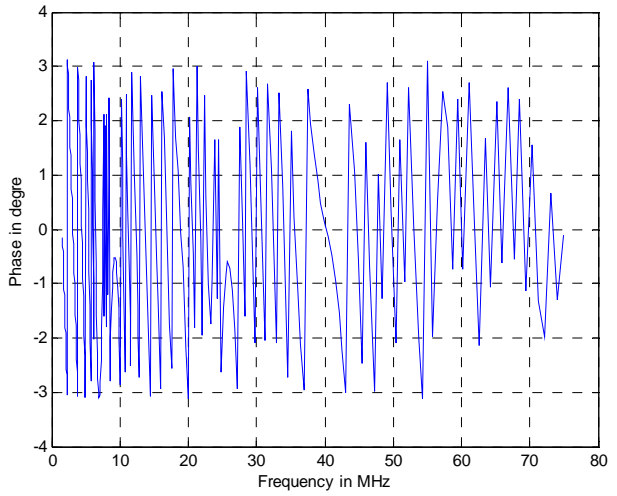

(b)

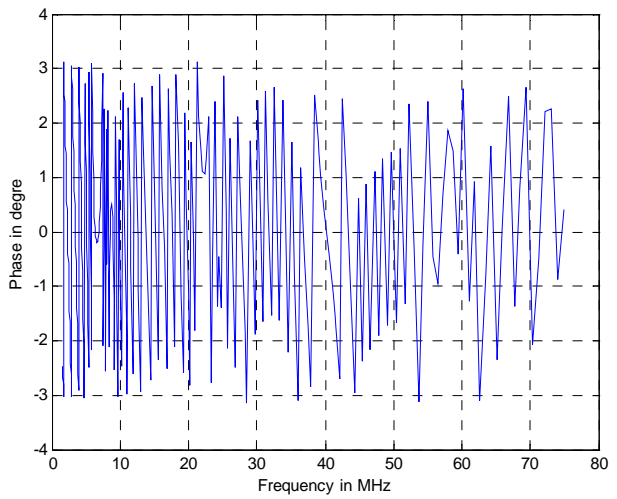

(d)

Figure 6. Phase response for PLC with 15 branches (a) 1 m; (b) 2 m; (c) 3 m; (d) 4 m. 


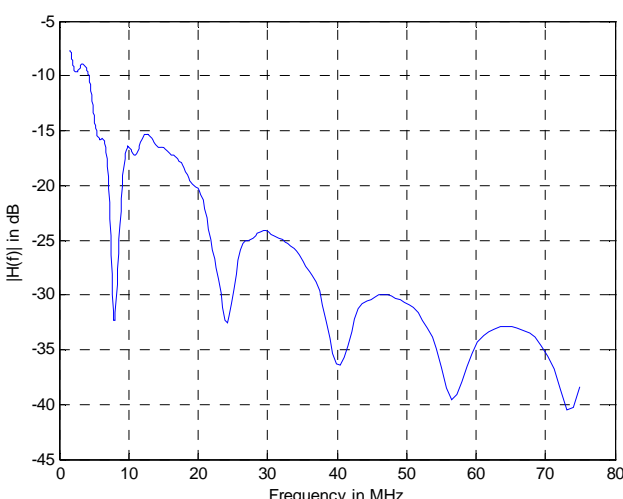

(a)

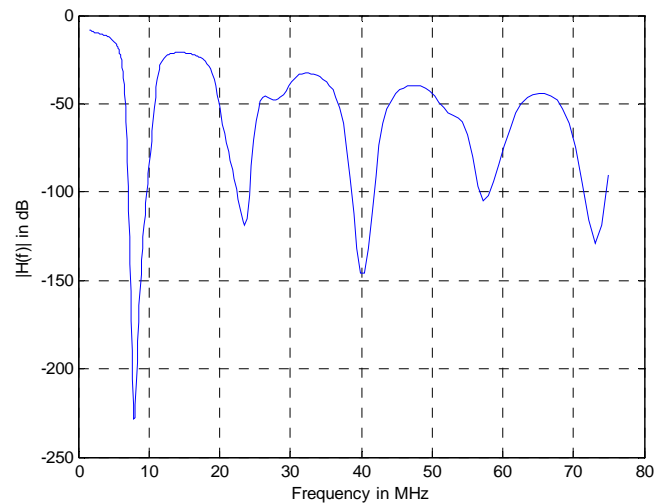

(c)

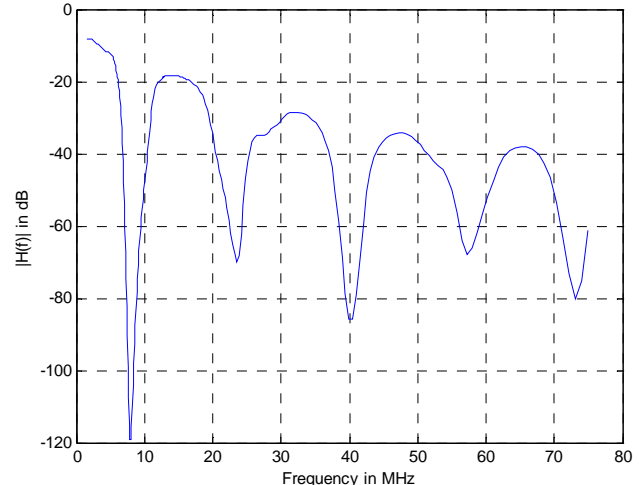

(b)

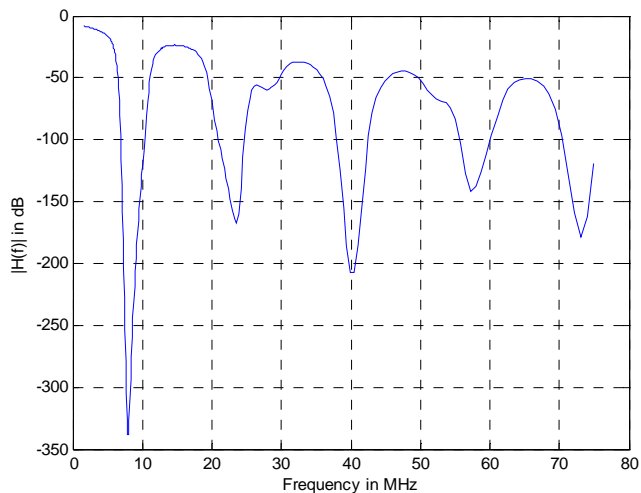

(d)

Figure 7. Simulation results for medium voltage channel with 1 branch (a); 5 branches (b); 10 branches (c); and 15 branches (d).

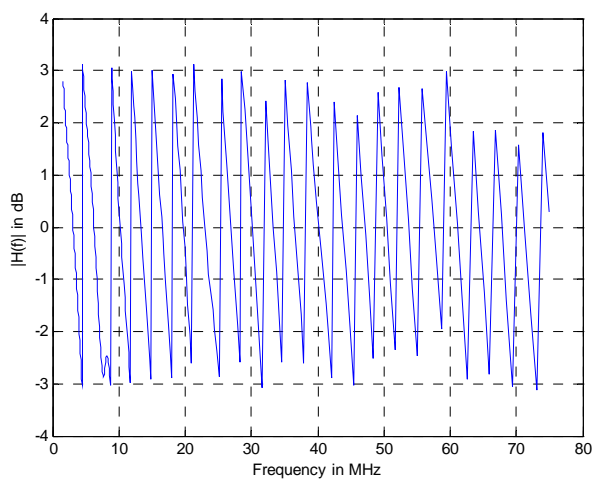

(a)

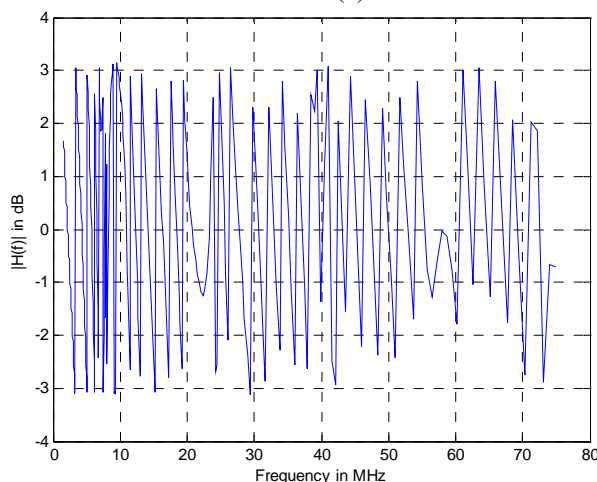

(c)

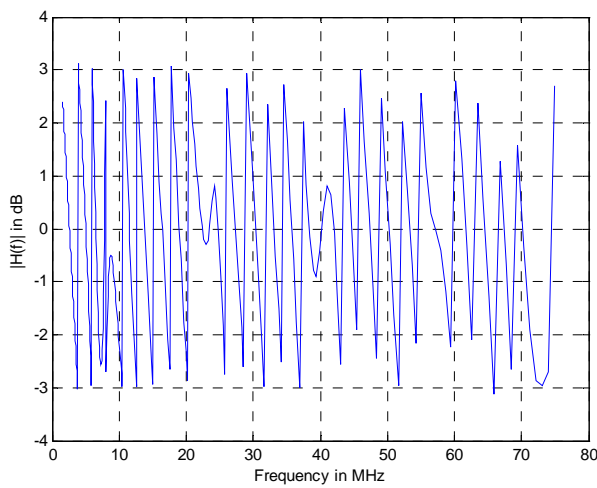

(b)

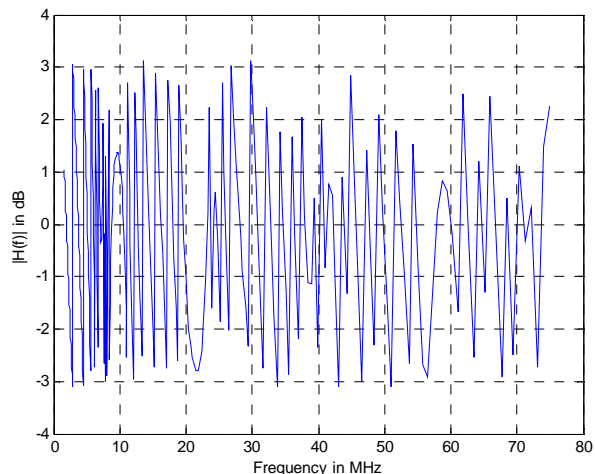

(d)

Figure 8. Phase response for medium voltage channel with 1 branch (a); 5 branches (b); 10 branches (c); and 15 branches (d). 
The peak points in the frequency responses tend to fluctuate as number of distributed branches increases.

The study of these phenomena is crucial for improving the performance of the PLC channel.

\section{REFERENCES}

[1] J. Ahola, "Applicability of Power-Line Communications to Data Transfer of On-Line Condition Monitoring of Electrical Drives," Ph.D Thesis, Lappeenranta University of Technology, Lappeenranta, 2003.

[2] T. Sartenaer and P. Delogne, "Deterministic Modelling of the (Shielded) Outdoor Powerline Channel Based on the Multiconductor Transmission Line Equations," IEEE Journal on Selected Areas in Communications, Vol. 24, No. 7, 2005, pp. 1277-1291.

[3] H. Philipps, "Modelling of Powerline Communications
Channels," Proceedings of 3rd International Symposium on Power-Line Communications and Its Applications, Lancaster, March 1999, pp. 14-21

[4] M. Zimmermann and K. Dostert, "A Multipath Model for the Powerline Channel," IEEE Transactions on Communications, Vol. 50, No. 4, 2002, pp. 553-559. doi:10.1109/26.996069

[5] T. Esmailian, et al., "In-Building Power Lines as High Speed Communication Channels: Channel Characterization and a Test Channel Ensemble," International Journal of Communication Systems, Vol. 16, No. 5, 2003, pp. 381400. doi:10.1002/dac.596

[6] I. C. Papaleonidopoulos, et al., "Evaluation of the TwoConductor HF Transmission-Line Model for Symmetrical Indoor Triple-Pole Cables," Measurement, Vol. 39, No. 8, 2006, pp. 719-728. 